\title{
Image analysis system for quantitative metallography
}

\author{
V K CHADDA, D G JOSHI, S N MURTHY, S V KULGOD, \\ C BABURAO*, D K BHATTACHARYA* and BALDEV RAJ*
}

Reactor Control Division, Bhabha Atomic Research Centre, Trombay, Bombay 400085, India * Radio Metallurgy Programme, Indira Gandhi Centre for Atomic Research, Kalpakkam 603102 , India

\begin{abstract}
This paper describes a Z-80 microprocessor-based image analyzer developed for global parameter evaluation of images over a $256 \times 256$ pixel frame. It consists of a microscope, CCD scanner, 6-bit video ADC, Z-80 computer and an image display monitor. Facilities are provided for feature erosion/dilation and halo correction. The paper also presents the details of another more powerful user microprogrammable HP1000 minicomputer-based image analysis system under development. This system consists of an optical microscope/epidiascope coupled to a chalnicon scanner. Here the $512 \times 512$ pixel image is acquired with 8-bit resolution. It provides for shading correction, auto-delineation, image processing and image analysis functions for evaluation of various basic and derived parameters.

Both the systems are software intensive and are realised according to requirements of quantitative metallography. They can also be used for analysis of images obtained in the fields of biology, medicine, geological survey, photography and space.
\end{abstract}

Keywords. Pixel; erosion; dilation; bit quad; global parameters; feature extraction; image analysis; metallography.

\section{Introduction}

Metallographic image analysis is essential for objective correlation of material properties with its microstructure. It is carried out by examining them under suitable electronic/optical instruments and analysing the image obtained.

A Z-80 microprocessor-based image analysis system with $256 \times 256$ pixel resolution has been developed indigenously. It provides estimation of different phases in microstructures through grey level detection (64 levels) and computation of their geometrical parameters.

An HP-1000 computer based image analysis system is also being developed with $512 \times 512$ pixel resolution incorporating facilities for image enhancement, auto-delineation, shading correction, light pen interaction and feature specific measurements.

\section{Z-80-based image analysis system}

A block diagram of the system is shown in figure 1 and described below:

\subsection{Imaging}

The image to be analyzed is obtained from samples observed under an optical microscope/epidiascope. It is projected on to the sensor array of a $\mathrm{CCD}$ video camera. 


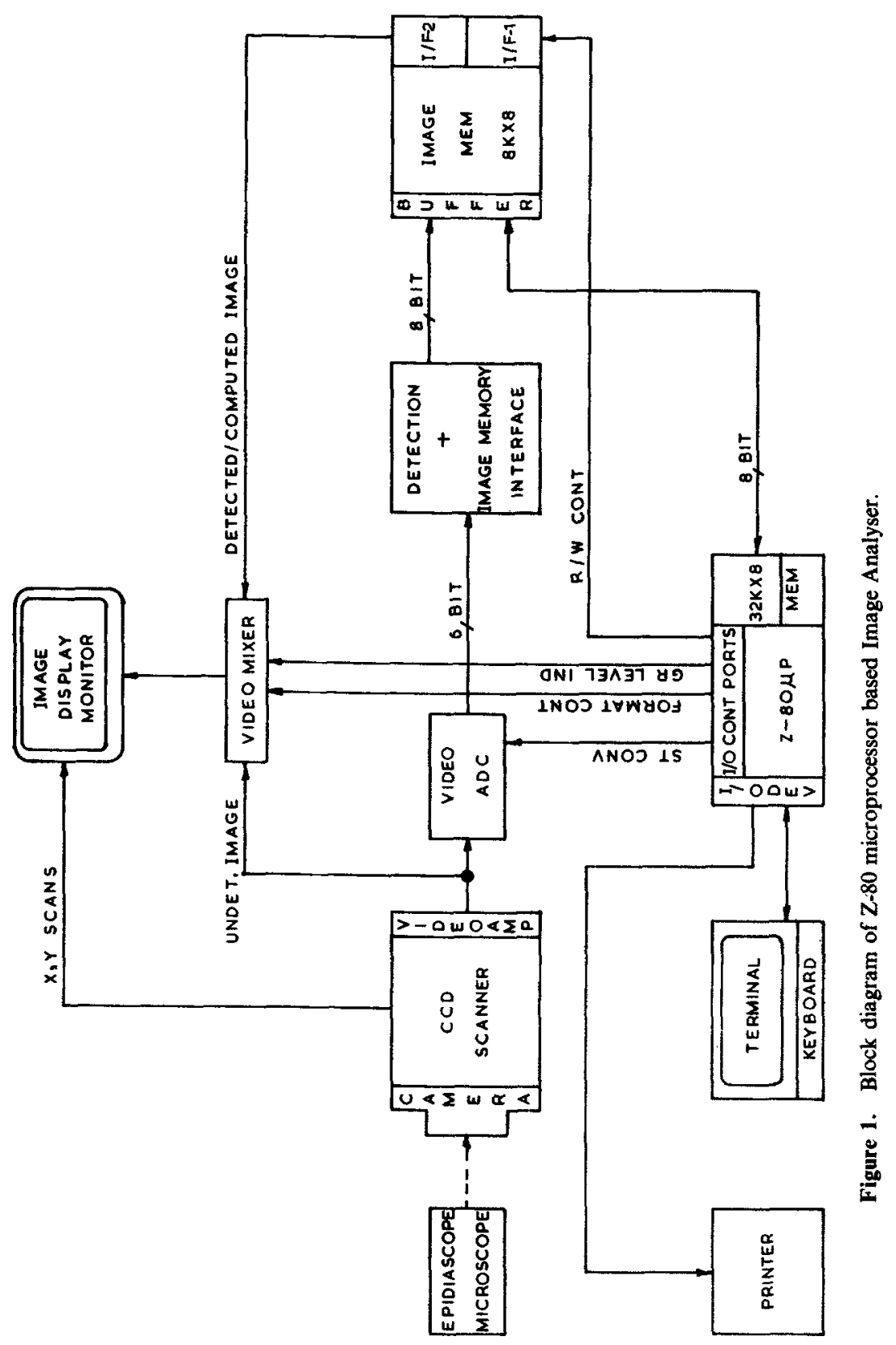


For image analysis 256 lines, 256 pixel matrix is selected. The remaining part of the frame is used to display a band of 64 grey levels and two cursors indicating the selected grey slice as shown in figure 2 .

\subsection{Data acquisition}

The scanner output is presented to a 6-bit video ADC for digitization. A simple scheme based on threshold detection was employed with upper and lower threshold levels. Detection constitutes obtaining a binary image (logic $0-1$ pattern) containing selected features. Logic ' 1 ' in the binary image corresponds to pixels lying in the selected grey slice and logic ' 0 ' for the background (Jenkinson 1982).

This binary image is compacted into byte format and stored into an $8 \mathrm{~K} \times 8$ bit memory for flicker-free image display and transferred to the computer memory for analysis.

\subsection{Data analysis}

Image analysis is carried out for evaluation of desired basic metallographic parameters on global basis. The system has facilities for erosion, dilation and halo error correction.

2.3a Lasic parameter evaluation: Here sets of $2 \times 2$ pixel patterns called bit quads are computed as shown in figure $3 \mathrm{a}$. Each pixel $A_{0}$ of the binary image is scanned and its associated bit quad $\left(A_{0}, A_{1}, A_{0}^{N}, A_{1}^{N}\right)$ is examined. These are represented as $C_{0}, C_{1}, \ldots C_{15}$ where $C_{1}=2^{3} A_{1}^{N}+2^{2} A_{0}^{N}+2^{1} A_{1}+2^{0} A_{0}$. At each pixel position one of the 16 combinations is encountered. A table is created in the memory to determine count of each $C_{i}$ over the entire frame. Based on combination of these $C_{i}$ values quad counts $Q_{1}, Q_{2}, Q_{3}, Q_{4}$ and $Q_{D}$ are computed as shown in figure $3 \mathrm{~b}$. Basic global parameters of an image over the entire frame are expressed in terms of the quad counts as follows (Pratt 1978).

$$
\begin{array}{ll}
A_{\text {(area) }} & =1 / 4\left(Q_{1}+2 Q_{2}+3 Q_{3}+4 Q_{4}+2 Q_{D}\right), \\
P_{\text {(perimeter) }} & =Q_{2}+0.707\left(Q_{1}+Q_{3}+2 Q_{D}\right), \\
E_{\text {(Euler count) }} & =1 / 4\left(Q_{1}-Q_{3}-2 Q_{D}\right) .
\end{array}
$$

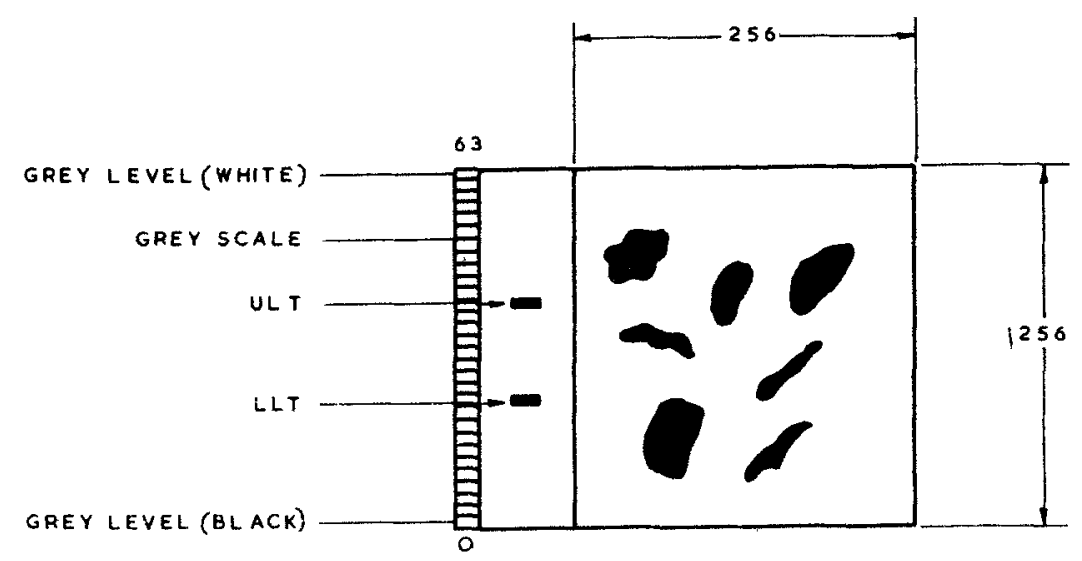

Figure 2. Format of image display on monitor. 
Feature count $C$ is related to Euler count by $E=(C-H)$ where $H=$ number of holes. If the number of holes is small in comparison with number of features, Euler number can be taken as a good estimate of feature count.

For horizontal projection $H_{p, 0-1}$ transition in the horizontal direction is observed as shown in figure $3 \mathrm{c}$ and $H_{p}$ is given by

$$
H_{p}=C_{2}+C_{6}+C_{10}+C_{14} \text {. }
$$

Similarly 0-1 transition in the vertical direction is observed as shown in figure $3 \mathrm{~d}$ and vertical projection $V_{p}$ is given by

$$
V_{p}=C_{4}+C_{6}+C_{12}+C_{14}
$$

Estimation of these parameters requires about 2.5 seconds.

2.3b Erosion and dilation: In the process of erosion, the touching objects are uniformly shrunk along their perimeters until a separation is achieved. Dilation is used to join separated parts of detected images.

For erosion and dilation, a reference pixel is considered in relation to its four nearest structuring elements as shown in figure $3 \mathrm{e}$. During erosion the reference pixel is

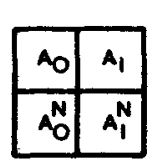

(a)

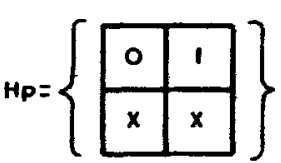

$c_{2}, c_{6}, c_{10}, c_{14}$

(c)

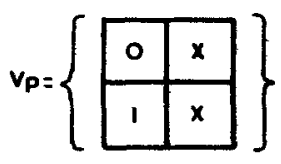

$c_{4}, c_{6}, c_{12}, c_{14}$

(d)

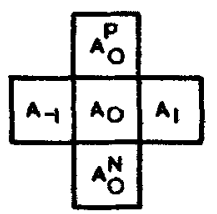

(e)

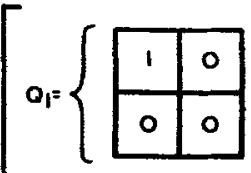

$c_{1}$

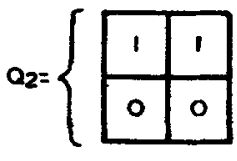

$c_{3}$

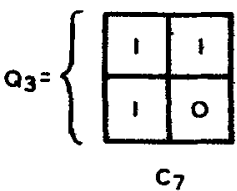

$c_{7}$

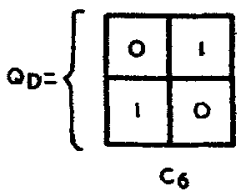

$c_{6}$

Figure 3. Image computation patterns. (a) Typical $2 \times 2$ pixel pattern. (b) Area, perimeter and feature count computation patterns. (c) Horizontal intercept pattern. (d) Vertical intercept pattern. (v) Structuring element for erosion and dilation. Note: $0=\operatorname{logic}$ ' 0 '; $1=\operatorname{logic}$ ' 1 '; $\mathbf{X}=$ don't care.

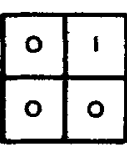

$c_{2}$

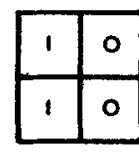

$\mathrm{C}_{5}$

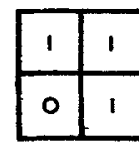

$c_{11}$

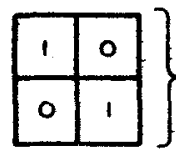

(b)

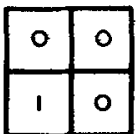

$C_{4}$

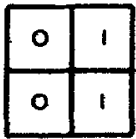

$c_{10}$

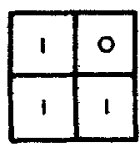

$c_{13}$

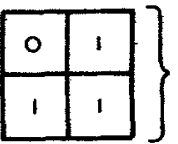

$c_{14}$
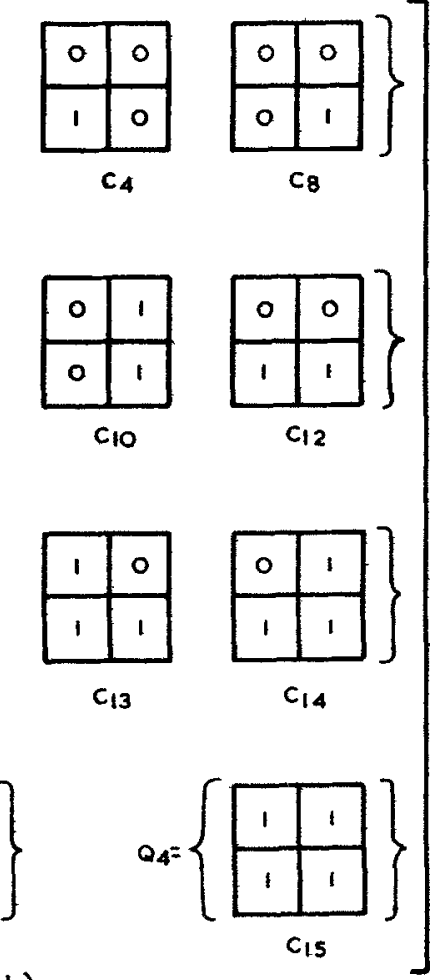

$\mathrm{Cg}_{\mathrm{g}}$

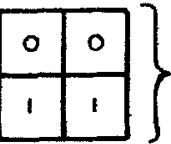

$C_{12}$ 
replaced by 'ANDing' of all the five pixels whereas dilation is accomplished by 'oring' of the same over the entire image frame. Time of execution for one step of erosion/dilation operation is about $0.7 \mathrm{sec}$.

2.3c Halo error correction: In a multiphase sample, when an intermediate phase is detected for computation using threshold level detection errors can occur due to finite system resolution. This has the effect of producing a 'halo' i.e. an apparent grey perimeter around a dark feature which is detected. Special algorithm has been developed based on repeated dilation and logical operations for halo error correction.

\subsection{Operator interaction}

Operator interaction with the system is accomplished under the control of a system monitor (Terrell 1978). A querry procedure guides the user step by step making the dialogue user friendly. Additionally, the monitor provides for execution of $1 / O$ operations like setting of undetected, detected and computed image display. Also there is a provision for print out of results in a standard format as shown in table 1.

\section{HP1000-based image processing and analysis system}

A powerful image processing and analysis system is being developed with $512 \times 512$ pixel resolution incorporating facilities such as shading correction, auto-delineation, image enhancement, light pen interaction and feature-specific measurements.

\subsection{Schematic details}

The system is based on a user microprogrammable HP1000-model 27 computer system. Figure 4 shows the block diagram of this system. Its imaging section consists of an optical microscope/epidiascope coupled to a chalnicon scanner. The video signal output is stored into a $512 \times 512 \times 8$ pixel frame grabber. Peripheral operations i.e. light pen interaction, autofocus, stage control, console scanning and image display monitor control are carried out by the Apple II computer. The image is transferred to HP1000 computer system via IEEE- 488 bus for processing and analysis.

\subsection{System software}

After shading correction and auto-delineation image processing and analysis functions for various basic and derived parameters are carried out in FORTRAN-77. Repetitive

Table 1. Micromet. (Radiometallurgy Programme, Kalpakkam)

\begin{tabular}{cccccccc}
\hline S1. No. & UT & LT & Count & Area & Peri & Vert & Horz \\
\hline 1 & 16 & 09 & 38 & 8402 & 2391 & 818 & 635 \\
2 & 38 & 26 & 86 & 19410 & 10017 & 3313 & 2732 \\
3 & 51 & 45 & 54 & 11692 & 3939 & 1391 & 945 \\
\hline
\end{tabular}

User: ABC; Specimen: Sensitized nuclear grade SS 316 sample.

Date: 19 January 1985; SP No. 1147. 


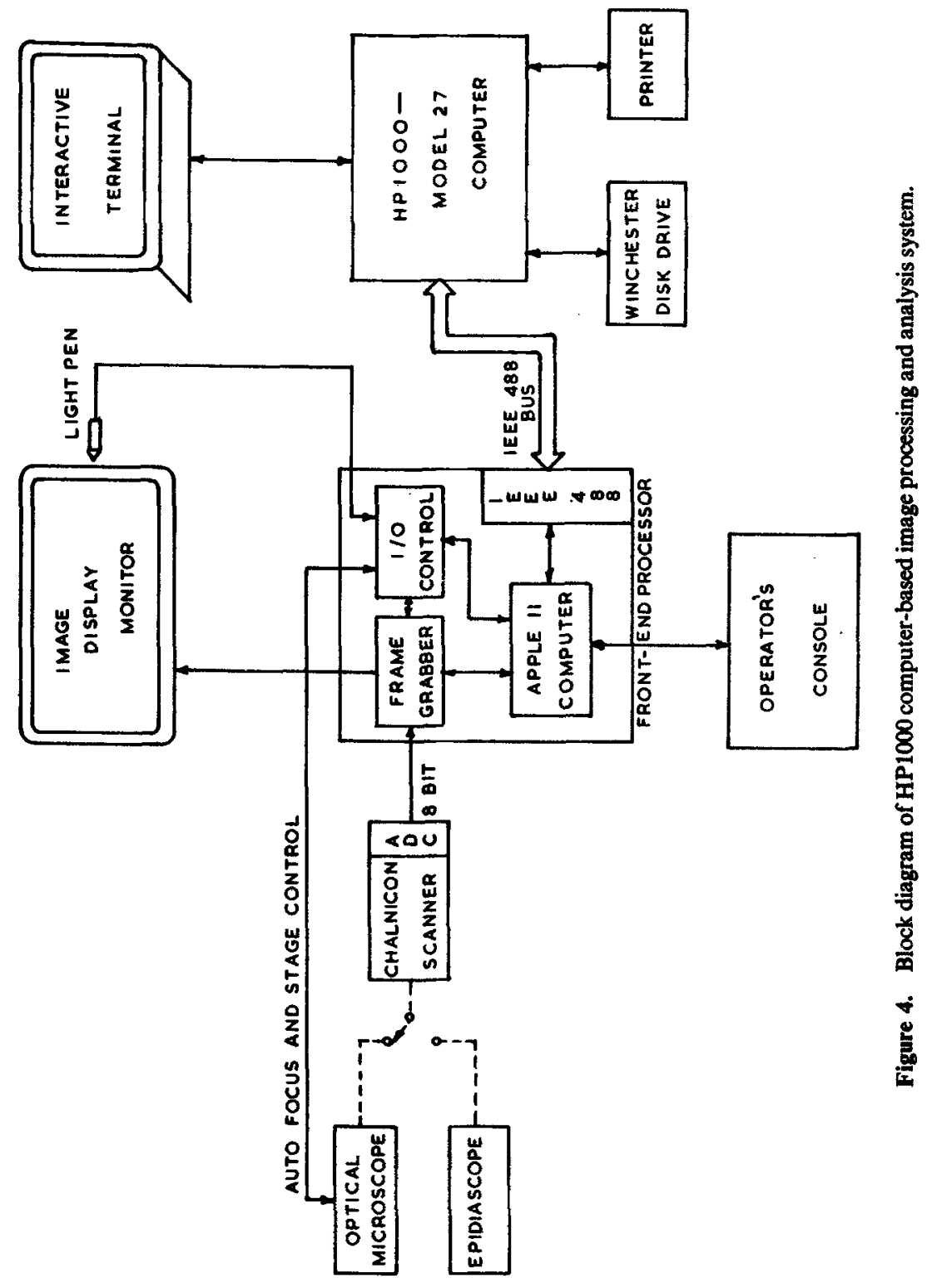


operations are microprogrammed for fast execution. Two specific schemes adopted for image analysis are continuous scan and features extraction methods.

3.2a Continuous scan method: Here parameters of each individual feature are measured separately. The detected binary image is continuously scanned. Whenever a feature is encountered it is indexed and the basic parameters are accounted for that feature. This technique provides accurate size distribution, spatial distribution and various derived parameters.

3.2b Feature extraction method: During evaluation of length, breadth and perimeter of a particular feature presence of other features interferes with the analysis. This can be avoided by using feature extraction method. In this technique, contour of the feature is traced. The complete feature is copied into another sector of memory and analyzed independently.

\section{Conclusion}

The Z-80 based image analyser is an inexpensive solution for measuring structural features in the image frame under view with limited resolution. It is a versatile tool for field-specific basic parameter evaluation. Provision exists for erosion/dilation and halo error correction. An HP1000 computer based image processing and analysis system is under development. The features being incorporated in this system are image enhancement, 2-D detection, shading correction, light pen interaction and featurespecific measurements. Both the systems being software intensive provide flexibility in selecting a particular operation and are open ended for further upgradation.

Though the image analysis is automated, the results obtained are still subjective. Human factors involved are in the preparation of samples/micrographs, selecting the desired features and in editing of images. Therefore, expert attention is indispensable for obtaining accurate results.

The systems are being realized taking into consideration the requirements of quantitative metallography. They are equally powerful for analysis of images obtained in the fields of photography, biology, medicine, geology, meteorology and space.

\section{Acknowledgements}

The authors are grateful to Dr P R Dastidar, Dr P Rodrigues and Sri S N Seshadri, for constant encouragement and providing facilities to develop the image analysis system.

\section{References}

Jenkinson G W 1982 Technical Exchange and Symposium on Image Analysis, University of Iron and Steel, Beijing

Pratt W K 1978 Digital image processing (New York: John Wiley \& Sons)

Terrell A C 1978 Microscope 2649 